\title{
Relación entre los estilos de aprendizaje y las teorías de enseñanza Relationship between learning styles and teaching theories
}

\author{
Rosana Pacheco Rios, Erasmo Maldonado Maldonado
}

Universidad Autónoma de Nuevo León, (México)

\begin{abstract}
Resumen. Este trabajo de investigación se realizó para saber la relación entre los estilos de aprendizaje con las teorías de enseñanza de los alumnos y de los docentes de la Facultad de Organización Deportiva (FOD) de la UANL. El método utilizado fue el deductivo, con un enfoque cuantitativo, realizando un estudio descriptivo-correlacional, transversal y de campo. Participaron 666 alumnos que corresponde al 64 \% de una población total de 1,037, con edades de 17 a 39 años y 59 docentes que corresponden al 84.2\% de un total de 70, de edades de 23 a 69 años. Los instrumentos de recolección de datos fueron: el «Cuestionario de Honey \& Alonso sobre estilos de aprendizaje» (CHAEA, 1992) y el TIEN (Teorías implícitas del profesor sobre la enseñanza, adaptado por Delgado y Zurita para ser aplicado en la Educación Física en el año 2003). Los resultados mostraron que la mayoría de los estilos de aprendizaje se relacionan con las teorías de enseñanza, exceptuando el estilo reflexivo. El estilo de aprendizaje activo se relaciona con la teoría de enseñanza tradicional, el teórico con la teoría de enseñanza expresiva o activa, el pragmático con la teoría de enseñanza emancipatoria o crítica, y el estilo reflexivo no se relaciona con ninguna teoría de enseñanza.
\end{abstract}

Palabras clave: estilos de aprendizaje, teorías de enseñanza, Educación Física.

Abstract. This research work was undertaken to inquire about the relationship between the learning styles and the teaching theories of the students and teachers of the School of Sports Organization of the Autonomous University of Nuevo Leon. The deductive method was used with a quantitative approach, undertaking a descriptive-correlational, cross-sectional, and field study. 666 students participated, which corresponds to 64\% of a total population of 1,037, with ages ranging from 17 to 38 years and 59 professors corresponding to 84.2\% of a total population of 70 , with ages ranging between 23 and 69 years. The data collection instruments used were the «Honey-Alonso Learning Styles Questionnaire» (CHAEA, 1992) and TIEN (Spanish acronym for Implicit Theories of the Professor Regarding Teaching, adapted by Delgado and Zurita to be implemented in the context of Physical Education in 2003). The results showed that most learning styles are related with teaching theories, with the exception of the reflective style, more specifically: the active learning style is related with the traditional teaching theory, the theoretical style relates to the expressive or active teaching theory, the pragmatic style relates with the emancipatory or critical teaching theory, and the reflective style didn't relate with any teaching theory.

Keywords: learning styles, teaching theories

\section{Introducción}

Uno de los mayores retos de la educación es lograr el desarrollo de competencias que permitan llevar a cabo una enseñanza y obtener un aprendizaje que puedan adaptarse a este mundo cambiante (Aguado, López y Hernández, 2017) y que conduzcan a docentes y estudiantes a ser productivos, innovadores y creativos. Todo proceso de enseñanza-aprendizaje en la formación universitaria requiere de un complejo constructo integrado por diferentes actores del sistema educativo (Serra, Muñoz, Cejudo, y Gil, 2017) dentro del cual debe tomarse en cuenta la diversidad de características presentes en el qué, cómo, cuándo, dónde, porqué, y para qué se enseña y se aprende.

Los estilos de aprendizaje unidos a diversas variables: expectativas y auto-concepto de los alumnos, entorno personal, contextual y social, habilidades sociales (Margalef, 2014; Núñez y Reyes, 2014), cognitivas y motoras, motivación, necesidades académicas, personales y del mercado laboral, experiencia y estrategias propias y aprendidas (Isaza, 2014) son definidos como mecanismos mediante los cuales la mente procesa y asimila la información (Gil, et al, 2007) de diferentes maneras con el fin de desarrollar el aprendizaje iniciando el proceso desde la forma de percibir (Gil, et al, 2007), analizar, decodificar, estructurar y generar esquemas y formas de comportamiento flexibles y cambiantes (Bahamón et al, 2012; Isaza, 2014); por ello es importante considerarlos en la construcción de procesos de enseñanza-aprendizaje (Madrid, Prieto, Samalot y Gil, 2016). Los estilos de enseñanza son considerados en la Educación Física (Fernández y Espada, 2017) como una herramienta metodológica importante porque son utilizados por los docentes para aportar conocimientos a los alumnos de una manera más eficaz, motivante e interesante (Shen \& Chen, 2007) lo cual da lugar a un aprendizaje más significativo, duradero (López y Moreno, 2002) y coherente (Ballesta, Izquierdo y Romero, 2011). Otros aspectos que influyen en las diversas formas de aprender y de enseñar son: el interés, los acervos cognitivos y motores, las características personales, el tem-

Fecha recepción: 09-07-15. Fecha de aceptación: 20-11-16 Rosana Pacheco Rios ross5559@hotmail.com peramento, el carácter, la personalidad, los valores culturales y sociales, la historia de vida, los conocimientos y las experiencias académicas, personales y contextuales.

Hay numerosas investigaciones que pretenden profundizar en la influencia que tienen los estilos de enseñar en el aprendizaje de los alumnos de la clase de Educación Física (Fernández y Espada, 2017) analizando diversas variables (sexo, edad, grado escolar, rendimiento académico, semestre que cursan -en los alumnos-y sexo, edad, años de experiencia, área curricular de la materia que imparten, grado académico obtenido, -en los docentes-). El propósito de este estudio es conocer si se relacionan las teorías de enseñanza con los estilos de aprendizaje.

Es importante considerar en forma crítica y analítica las características, los principios psicopedagógicos, las experiencias provenientes de la investigación y la trascendencia de los productos obtenidos de las experiencias del aprendizaje y de la enseñanza mediante la investigación-acción para detectar las disposiciones, preferencias, tendencias, patrones de conducta, habilidades, fortalezas y áreas de oportunidad, actuales de maestros y estudiantes, como datos importantes que fundamenten el diseño, re-diseño, adecuación, planeación, organización o implementación de estrategias, métodos, técnicas, actividades y tareas con una pedagogía y didáctica apropiada, para llevar a cabo las funciones correspondientes con conocimiento, integridad, eficacia, eficiencia, ética, seguridad, control, calidad y con la adecuada organización de los recursos (humanos, materiales, tecnológicos y tiempo) con los que se cuente.

\section{Material y Método}

En este estudio se aplicó un método deductivo, con un enfoque cuantitativo, realizando un estudio descriptivo-correlacional, transversal y de campo, para detallar en forma objetiva los estilos de los estudiantes y las teorías de enseñanza de los docentes, haciendo comparaciones entre las variables correspondientes a cada instrumento y analizando las relaciones entre los estilos y las teorías.

\section{Población alumnos}

Participaron 666 alumnos (el 64\% de la población) de un total de 1,037 con edades de 17 a 39 años $(M=20.12, D T=2.30)$. La obtención 
de la muestra se realizó calculando el nivel de confianza cuyo resultado fue $=.99$, con un margen de error $=.03$, realizando este procedimiento con la calculadora estadística de muestreo de http:// www.pwpamplona.com/wen/calcu/calculadora1.htm. Los resultados mostraron que el tamaño de la muestra adecuada es de 664 (y se aplicó a 666 alumnos menos un cuestionario que fue eliminado quedando 665), con una proporción a priori de .50.

\section{Instrumento de recolección de datos de los alumnos}

El instrumento de recolección de datos es el «Cuestionario de Honey \& Alonso sobre estilos de aprendizaje (1992)» (CHAEA), que adaptaron al contexto español y deriva del LSQ (Learning Style Questionare) cuyos autores son Honey \& Mumford (1986). Este cuestionario fue elegido porque es uno de los instrumentos mayormente utilizados para medir los estilos de aprendizaje. En Iberoamérica ha sido usado en diversas investigaciones desde 1992 (García, 2006); está compuesto por 80 ítems con respuestas dicotómicas, eligiendo con signo (+) si se está de acuerdo con el ítem, o con un signo (-) si se está en desacuerdo con este último. Los 80 ítems están clasificados en cuatro grupos de 20 ítems en cada uno, los cuales corresponden a cuatro Estilos deAprendizaje: Activo, Reflexivo, Teórico y Pragmático (tabla 1).

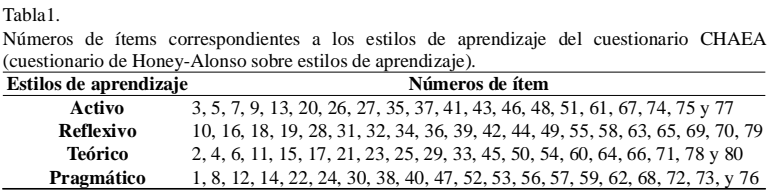

El procedimiento para recabar los datos fue el siguiente: se realizó una calendarización para aplicar el cuestionario a los alumnos de $1^{\circ}$ a $8^{\circ}$. semestre. El cuestionario se aplicó en los salones de clase por grupo y semestre. La información contenida en el instrumento se trató de la siguiente manera: los datos de cada una de las variables del cuestionario se vaciaron en el SPSS versión 16. Para conocer la validez y la fiabilidad del CHAEA se realizaron los siguientes análisis y pruebas: a) Prueba de fiabilidad mediante el coeficiente Alpha de Cronbach, el cual puede tomar valores entre cero y uno, donde cero significa confiabilidad nula y uno representa confiabilidad total. Este análisis se llevó a cabo sacando la varianza de los ítems y del puntaje total y mediante la matriz de correlación de los ítems con el programa estadístico SPSS versión 16.b) Análisis factorial confirmatorio (AFC) de los 20 ítems de cada uno de los cuatro estilos en forma separada, mediante el modelo estadístico de ecuaciones estructurales utilizando el Programa estadístico LISREL 8.8. c) Análisis factorial exploratorio y confirmatorio de los cuatro estilos de aprendizaje en forma separada, eligiendo 4 ítems de cada estilo aleatoriamente para poner a prueba un total de cinco modelos hipotéticos. Y para evaluar el ajuste de cada modelo hipotético con cada uno de los modelos teóricos y la matriz de datos recogida tanto del AFC como del SEM (error estándar), se utilizaron los siguientes índices: $c^{2}, c^{2} / g l$, NNFI(Non Normed Fit Index), CFI(Comparative Fit Index), y RMSEA (Root Mean Square Error of Approximation) y finalmente, se realizaron análisis descriptivos de comparación mediante pruebas no paramétricas utilizando el Método de U de Mann-Witney, utilizado para comparar dos muestras independientes, y el Método de Kruskal Wallis utilizado cuando se comparan más de dos muestras independientes. Dichos análisis se efectuaron con el programa estadístico SPSS versión 16.

\section{Población docente}

También participaron 59 docentes que corresponden al 84.2\% de un total de 70 , de edades de 23 a 69 años $(M=48.88 D T=11.82)$ que imparten clases de nivel Licenciatura en la Facultad de Organización Deportiva de la Universidad Autónoma de Nuevo León y que están oficialmente registrados y asignados para llevar a cabo los cursos presenciales de $1^{\circ}$ a $8^{\circ}$. semestre. Para la obtención de la muestra se realizó un cálculo mediante la fórmula que aparece en http:// www.pwpamplona.com/wen/calcu/calculadora1.htm utilizada para conocer el tamaño muestral para la proporción. Los datos que se ingresaron en este formato fueron los siguientes: tamaño de la población total $=70$; nivel de confianza $=.94$; margen de error $<0$ =. 05 . Los resultados mostraron que el tamaño de la muestra es de 59, con una proporción a priori de.50.

\section{Instrumento de recolección de datos de los docentes}

El instrumento de recolección de datos es el TIEN (Teorías implícitas del profesor sobre la enseñanza que proporciona datos sobre el estilo personal de enseñar cuyos autores e instrumento original son: Rodrigo, Rodríguez \& Marrero, 1993; que se basaron en las aportaciones de Piaget con el instrumento denominado: «Las teorías implícitas». Una aproximación al conocimiento cotidiano). Este cuestionario fue adaptado para ser aplicado en el área de la Educación Física por Delgado y Zurita (2003). Consta de 26 ítems, que se responden con una escala tipo likert de cero a siete, que oscila desde cero, «no se está de acuerdo», hasta «totalmente de acuerdo» siete. Los 26 ítems forman una estructura de cinco grupos los cuales corresponden a cada uno de los estilos de enseñanza: dependiente o tradicional, productiva o tecnológica, expresiva o activa, interpretativa o constructivista y emancipadora o crítica (tabla 2).

\begin{tabular}{l} 
Tabla 2. \\
Números de ítems del cuestionario TIEN, correspondientes a cada teoría de enseñanza. \\
\hline \multicolumn{1}{c}{ Teoría de enseñanza } \\
\hline Dependiente o tradicional
\end{tabular}

El procedimiento para recabar los datos fue el siguiente: se realizó una calendarización para aplicar el cuestionario a los docentes en sus áreas de trabajo, respetando su tiempo disponible para ello. La información contenida en el instrumento se trató de la siguiente manera. Se vaciaron los datos da cada una de las variables contenidas en el «Cuestionario TIEN» de los docentes en el programa estadístico SPSS versión 16. Se realizó un análisis factorial de fiabilidad. Se calcularon los índices de tipicidad y la polaridad para saber qué teoría de la enseñanza predomina y su relación con el resto de teorías (polaridad). Posteriormente, se hicieron comparaciones para dos muestras independientes con la prueba de $t$ de Student, mediante el método estadístico ANOVA utilizado para comparar más de dos muestras independientes y mediante la prueba de post-hoc de Scheffe, debido a la distribución normal de los datos señalada por la prueba de Shapiro-Wilk.

Una vez realizados los análisis anteriores se procedió a estimar la relación entre las teorías de enseñanza y los estilos de aprendizaje por medio de tablas de contingencia, las cuales son usadas para registrar y analizar la relación entre dos o más variables habitualmente de naturaleza cualitativa y cuando las opciones de respuesta son dicotómicas. En tal análisis se consideraron los valores de $x^{2}$, coeficiente Phi, la V de Cramer y el coeficiente de contingencia C. En este sentido, El coeficiente Phi es una medida para la intensidad de la relación entre variables dicotómicas. El coeficiente de contingencia de Pearson expresa la intensidad de la relación entre dos (o más) variables nominales u ordinales (prueba de chi cuadrado de Pearson).

\section{Resultados de los alumnos}

Descripción de los datos demográficos de la muestra de estudio (tabla 3).

Tabla 3.
\begin{tabular}{ccccc} 
Datos demográficos sobre sexo y edades de los alumnos. \\
\hline Edad & Sexo & $\boldsymbol{n}$ & $\boldsymbol{M}$ & $\boldsymbol{D}$ \\
\hline Mínima y Máxima & Hombres & 479 & 20.31 & 2.37 \\
17 y 39 & Mujeres & 186 & 19.60 & 2.01 \\
& & Total: 665 & 20.12 & 2.30 \\
\hline
\end{tabular}

\section{Análisis de fiabilidad del Cuestionario CHAEA}

Los resultados de consistencia interna de las escalas se muestran en la tabla 4. En dicho análisis se manejaron los siguientes criterios: a) una 
correlación inter-ítem entre $\mathrm{r}=.20$ y r $=.70$; y b) un mínimo de correlación ítem total corregida de r =.30 (Kidder \& Judd, 1986). Además los ítems fueron analizados comprobando si el alfa de la escala aumentaba con la eliminación de algúnítem.

\begin{tabular}{|c|c|c|c|}
\hline & & & \\
\hline Estilo & $\alpha$ & $\begin{array}{l}\text { Ítems que saturan por debajo de } \mathbf{3 0} \\
\text { (no son compatibles con lo que miden) }\end{array}$ & $\begin{array}{l}\text { Ítem eliminado para } \\
\text { que aumente } \alpha\end{array}$ \\
\hline Activo & .60 & $7,13,20,26,37,41,46,43,51,61,67,75$ y 74 & 9 \\
\hline Reflexivo & .60 & $16,32,34,36,39,42,49,55,58,65,70$ у 79 & 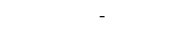 \\
\hline Teórico & .59 & $2,6,11,15,21,23,25,29,33,45,54,60,64$ у 80 & - \\
\hline Pragmático & .50 & $2,6,11,15,21,23,25,29,33,45,54,60,64$ y 80 & - \\
\hline
\end{tabular}

Estos resultados muestran que este instrumento de medida es poco confiable, por tanto se sugiere eliminar algunos ítems, como aquellos que saturan por debajo de $p<0,30$.

\section{Análisis factorial confirmatorio del cuestionario CHAEA}

Partiendo de una muestra efectiva de 666 estudiantes y de un alejamiento de la normalidad de los datos, se realizó el AFC con el método robusto de Máxima Verosimilitud siguiendo las recomendaciones de Chou, Bentler \& Satorra (1991).

Resultados del análisis factorial confirmatorio de los estilos de aprendizaje (con todos los ítems) que muestra los índices de ajuste de cada factor analizado por separado (tabla 5).

Tabla 5.

Análisis factorial confirmatorio de los estilos de aprendizaje con todos los ítems.

\begin{tabular}{|c|c|c|c|c|c|c|c|}
\hline Estilos & $x^{2}$ & $g . l$ & $p$ & RMSA & CFI & NNFI & $\begin{array}{l}\text { Ítems que saturan } \\
\text { por debajo de .40 }\end{array}$ \\
\hline Activo & 365.28 & 170 & $<<.001$ & .042 & .943 & .937 & 43 y 61 \\
\hline Reflexivo & & & & No $\mathrm{Cc}$ & iverge & & \\
\hline Teórico & 269.97 & 170 & $<.001$ & .031 & .974 & .971 & $2,23,25$ y 80 \\
\hline Pragmático & 375.82 & 170 & $<.001$ & . 044 & .920 & .911 & $14,76,72, y 62$ \\
\hline
\end{tabular}

Esta tabla muestra los índices de ajuste de cada factor analizado por separado dado el tamaño de la muestra, el cual no fue suficiente para analizar todos los factores que componen el cuestionario en su conjunto. En ella se puede observar que los modelos para cada factor por separado ajustan los datos con los modelos (son mayores a .010 para CFI y NNFI), sin embargo hay ítems que tienen baja saturación factorial y que no fue significativa ( $\mathrm{t}<1.96, \mathrm{p}>.05$ ), además el factor reflexivo exhibe problemas, siendo esto un indicio para su eliminación.

Por tanto se llevó a cabo un segundo AFC eliminando aquellos ítems cuyo peso factorial no fue significativo para el factor del cuestionario. Los resultados aparecen en la tabla 6.

Tabla 6.

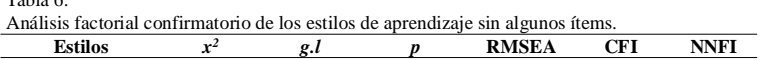

\begin{tabular}{ccccccc} 
Estilos & $\boldsymbol{X}^{2}$ & $\boldsymbol{g . I}$ & $\boldsymbol{p}$ & RMSEA & CFI & NNFI \\
\hline Activo & & & & & & \\
Reflexivo & - & 135.44 & $<.001$ & .034 & .966 & .962 \\
Teórico & 141.37 & - & - & - & - & - \\
Pragmático & 228.28 & 104 & $<.008$ & .024 & .989 & .987 \\
\hline
\end{tabular}
den

Análisis factorial confirmatorio sin los ítems que no correspon-

Resultados del análisis factorial confirmatorio de los estilos de aprendizaje eliminando los ítems cuyo peso factorial no fue significativo (tabla 6).

EsteAFC eliminando los ítems 43 y 61 del estilo activo sugiere que el resto de los ítems tienen un peso significativo en este factor $(\mathrm{t}>1.96$, $p<.05$ ) para el estilo pragmático (eliminando los ítems 14, 62, 72 y 76) y el estilo teórico (eliminando los ítems 2, 23, 25 y 80) todos los demás ítems logran saturar significativamente $(\mathrm{t}>1.96, p<.05)$ en los tres modelos con la eliminación de ítems (tabla 6), las diferencias de los índices de ajuste incrementales con respecto a los modelos con todos los ítems (tabla 5) son mayores a .010 para CFI y NNFI, con lo que se aprueba que los modelos con la eliminación de los ítems se ajustan más a los datos.

Dado que el instrumento mide los cuatro estilos de aprendizaje en conjunto, siguiendo el estudio Richaud, (2005b) se evaluó la estructura del instrumento considerando sus cuatro factores en cinco modelos distintos los cuales agrupan cuatro ítems en cada uno, dispuestos al azar (tabla 7). Los resultados aparecen en la tabla 14.

Análisis factorial confirmatorio agrupando cada estilo de aprendizaje en 5 modelos aleatorios

Agrupación de los ítems de cada uno de los estilos de aprendizajeen 5 Modelos aleatorios (tabla 7).

Tabla 7.
Ítems que conforman los estilos de aprendizaje para los modelos aleatorios puestos a prueba.
\begin{tabular}{ccccccc}
\multicolumn{6}{c}{ M o d e I o s } \\
a l e a t o r i o s \\
Estilos & $\mathbf{1}$ & $\mathbf{2}$ & $\mathbf{3}$ & $\mathbf{4}$ & $\mathbf{5}$ \\
\hline Activo & $13,67,9,51$ & $5,20,75,26$ & $7,27,74,35$ & $77,48,3,46$ & $37,41,43,61$ \\
\hline Reflexivo & $19,32,70,18$ & $10,58,79,34$ & $16,28,42,49$ & $44,63,69,31$ & $36,39,55,65$ \\
Teórico & $21,50,80,15$ & $6,33,60,45$ & $11,29,54,64$ & $4,17,71,78$ & $2,23,66,25$ \\
Pragmático & $40,59,73,38$ & $8,24,47,68$ & $12,30,57,56$ & $1,52,53,22$ & $14,62,72,76$ \\
\hline
\end{tabular}

Esta tabla muestra que los ítems que no corresponden al estilo que miden son el número 20 correspondiente al estilo activo y el ítem 8 correspondiente al estilo pragmático, de acuerdo con el modelo aleatorio 2 .

Resultados del análisis factorial confirmatorio realizado a cada uno de los modelos aleatorios en los que fueron agrupados los estilos de aprendizaje (tabla 8).

\begin{tabular}{|c|c|c|c|c|c|c|}
\hline Modelo & $x^{2}$ & g.I & $p$ & RMSEA & NNFI & CFI \\
\hline 1 & 128.59 & 98 & .02 & .022 & .982 & .985 \\
\hline 2 & 152.71 & 98 & $<.01$ & .030 & .93 & .94 \\
\hline 3 & 223.40 & 98 & $<.01$ & .045 & .887 & .907 \\
\hline 4 & \multirow{2}{*}{\multicolumn{6}{|c|}{ No converge }} \\
\hline 5 & & & & & & \\
\hline
\end{tabular}

En el modelo 2 los ítems 8 y 20 no saturan significativamente en el factor del estilo de aprendizaje hipotetizado, mientras que los modelos 1,2 y 3 , algunos de los factores latentes correlacionan altamente, (por encima de 85), con lo que se pone en duda la validez discriminante y se descartan dichas estructuras factoriales. En cuanto al $5^{\circ}$. Modelo, no converge (es decir el software no lo puede calcular). Finalmente, el $4^{\circ}$. Modelo, además de presentar un buen ajuste, todos los ítems saturan de forma significativa $(p<.05)$ y la relación entre los factores latentes aprueba la validez discriminante (Tabla 9).

Resultados del cuarto modelo aleatorio producto de la correlación de la matriz Phi entre los factores latentes (tabla 9).

\begin{tabular}{|c|c|c|c|}
\hline \multicolumn{4}{|c|}{ Modelo 4} \\
\hline Estilos & Activo & Reflexivo & Teórico \\
\hline Reflexivo & $-.53 * *$ & & \\
\hline Teórico & $-.73 * *$ & $.86^{* *}$ & \\
\hline Pragmático & $.23 * *$ & $.14^{* * *}$ & $.06 * *$ \\
\hline
\end{tabular}

De tal forma que los resultados obtenidos del cuarto modelo aleatorio indican que esta es la estructura más adecuada para medir los estilos de aprendizaje. Por ello dicho modelo es el que se utiliza en los análisis siguientes de esta investigación.

\section{Análisis descriptivo de los estilos de aprendizaje}

Para caracterizar los estilos de aprendizaje de los alumnos de la Facultad de Organización Deportiva que cursan materias de primero a octavo semestre, de nivel Licenciatura de la Universidad Autónoma de Nuevo León, México. Se analizaron los descriptivos tomando como referencia el instrumento resultante del $4^{\circ}$. Modelo (tabla 10).

Resultados del estilo de aprendizaje predominante considerando el $4^{\circ}$. Modelo Aleatorio (tabla 10).

Tabla 10.

\begin{tabular}{|c|c|c|c|c|}
\hline \multicolumn{5}{|c|}{ Resultados con todos los ítems } \\
\hline Estilo de aprendizaje & $M$ & $D T$ & asimetría & curtosis \\
\hline Activo & 8.359 & 3.606 & .779 & .235 \\
\hline Reflexivo & 8.968 & 3.672 & .752 & -.083 \\
\hline Teórico & 7.853 & 3.973 & .752 & -.295 \\
\hline Pragmático & 9.575 & 3.404 & .602 & -.144 \\
\hline \multicolumn{5}{|c|}{ Resultados con el $4^{\circ}$. Modelo Aleatorio } \\
\hline Activo & .468 & .313 & .069 & -1.020 \\
\hline Reflexivo & .733 & .272 & -.769 & -.303 \\
\hline Teórico & .682 & .287 & -.651 & -.454 \\
\hline Pragmático & .822 & .231 & -1.369 & 1.651 \\
\hline
\end{tabular}

Los resultados de esta tabla muestran que el estilo de aprendizaje predominante es el pragmático y el que menos predomina es el activo. 


\section{Resultados de los docentes}

Para obtenerlos se realizó un análisis de fiabilidad, se calcularon los índices de tipicidad y de polaridad de las teorías de la enseñanza siguiendo los estudios llevados a cabo por Delgado \& Zurita, (2003) considerando todos los ítems del cuestionario y sin considerar los ítems, cinco, siete, 11 y 24 que fueron eliminados como resultado del análisis de fiabilidad mediante el método de Alpha de Cronbach mediante el cual se analiza cada teoría de enseñanza demostrando que sólo el factor expresivo o activo tiene adecuada fiabilidad (.72), para el resto de los factores los resultados de fiabilidad fueron inadecuados (Dependiente o tradicional con unAlpha de .532, sugiriendo eliminar el ítem 5 , para que el valor de alfa suba a .60; productiva o tecnológica con un Alpha de .57; interpretativa o constructivista con unAlpha de .39 , sugiriendo eliminar el ítem 11 para que la fiabilidad aumente a .49; y emancipatoria o crítica con un Alpha de .56 sugiriendo eliminar los ítems siete y 24, para que la fiabilidad aumente.66.

Nota: coeficientes con Alphas de 0.60 pueden ser considerados aceptables en el caso de escalas conformadas por un bajo número de ítems (Hair, Babin, Anderson \& Tatham, 2006), tal es el caso de los factores critico y dependiente tras la eliminación de los ítems sugeridos en el análisis de fiabilidad. El factor productivo, dada su importancia teórica y cercanía al valor mínimo de .60 se ha dejado para el posterior análisis de los datos.

\section{Análisis descriptivo de las teorías de enseñanza}

Para caracterizar las teorías de enseñanza de los docentes de la Facultad de Organización Deportiva que imparten materias de primero a octavo semestre de nivel Licenciatura de la Universidad Autónoma de Nuevo León, México, se calculó la tipicidad y la polaridad para saber qué teoría de la enseñanza predomina (Delgado \& Zurita, 2003), considerando todos los ítems del cuestionario y sin considerar los ítems, cinco, siete, 11 y 24. Los resultados que derivan de los cálculos de la tipicidad y de la polaridad muestran cuáles son las teorías más significativas, así como la pertenencia o no de los sujetos o de los grupos a éstas. Los valores asignados a los índices de tipicidad y polaridad se describen en la tabla 11.

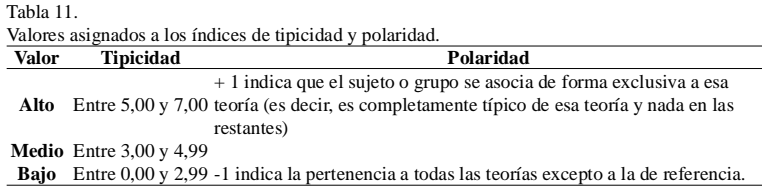

Para comprobar la suma de todas las polaridades debe ser =cero. Los resultados (+) son los más altos y los resultados (-) son los más bajos.

Resultados de la teoría de enseñanza predominante de los docentes de la FOD de la UANL (tabla 12).

Tabla 12.

Teorías de enseñanza de los profesores que predominan, con todos los ítems y quitando los ítems 5, 7, 11 y 24.

\begin{tabular}{|c|c|c|c|c|c|c|}
\hline \multicolumn{7}{|c|}{ Teoría de enseñanza que predomina en los docentes } \\
\hline \multirow{2}{*}{$\begin{array}{l}\text { Teoría de ens } \\
\text { Teorías de enseñanza }\end{array}$} & \multicolumn{3}{|c|}{ con todos los ítems } & \multicolumn{3}{|c|}{ sin los ítems 5, 7, 11 y 24} \\
\hline & Tipicidad & $D T$ & Polaridad & Tipicidad & $D T$ & Polaridad \\
\hline te o tradici & 4.5661 & 1.08726 & -.0324 & 4.6102 & 1.18521 & .0165 \\
\hline Expresiva o Activa & 6.2684 & 80484 & 2716 & 6.2684 & 80484 & 3126 \\
\hline Productiva o Tecnológi & 4.4932 & 1.02751 & -.0454 & 4.4932 & 1.02751 & -.0043 \\
\hline Interpretativa o Constructivista & 5.1737 & .99270 & .0761 & 5.1130 & 1.18356 & .1063 \\
\hline Emancipadora o Crítica & 3.2362 & 1.05666 & -.2699 & 2.1031 & 1.44141 & -.4312 \\
\hline
\end{tabular}

Los resultados de esta tabla (tomando en cuenta todos los ítems y sin los ítems eliminados), muestran que la teoría de enseñanza que predomina es la expresiva o activa y la menos predominante es la emancipadora o crítica.

Análisis estadístico para conocer la relación que hay entre los Estilos de Aprendizaje y las Teorías de Enseñanza, analizando los datos con todos losítems

Resultado de la relación del estilo de aprendizaje activo con las teorías de enseñanza (tabla 13).

\begin{tabular}{|c|c|c|}
\hline Estilo de aprendizaje & Análisis estadístico & Teoría de enseñanza \\
\hline \multirow{5}{*}{ Activo } & $\mathrm{X}^{2}=.042$ & \multirow{4}{*}{ Dependiente o tradicional } \\
\hline & Phi $=1.6$ & \\
\hline & $\mathrm{U}=.68$ & \\
\hline & $\mathrm{Cc}=.86$ & \\
\hline & $\mathrm{X}^{2}==.05$ & \multirow{4}{*}{ Productiva o tecnológica } \\
\hline \multirow[t]{3}{*}{ Activo } & $\begin{array}{c}\text { Phi }=1.5 \\
\mathrm{U}=.63\end{array}$ & \\
\hline & $\mathrm{Cc}=.84$ & \\
\hline & $\mathrm{X}^{2}==.05$ & \\
\hline \multirow{4}{*}{ Activo } & Phi $=1.18$ & \multirow{4}{*}{ Expresiva o activa } \\
\hline & $\mathrm{U}=.48$ & \\
\hline & $\mathrm{Cc}=.76$ & \\
\hline & $\mathrm{X}^{2}==.05$ & \\
\hline \multirow[t]{4}{*}{ Activo } & $\mathrm{Phi}=1.2$ & \multirow[t]{3}{*}{ Interpretativa o constructivista } \\
\hline & $\mathrm{U}=.52$ & \\
\hline & $\mathrm{Cc}=.78$ & \\
\hline & $\mathrm{X}^{2}==.05$ & \multirow{3}{*}{ Emancipadora o crítica } \\
\hline \multirow[t]{2}{*}{ Activo } & Phi $=1.4$ & \\
\hline & $\begin{array}{l}\mathrm{U}=1.57 \\
\mathrm{C}_{\mathrm{C}}=81\end{array}$ & \\
\hline
\end{tabular}

Los resultados de esta tabla muestran que sólo el estilo de aprendizaje activo se relaciona con la teoría de enseñanza dependiente o tradicional.

Resultado de la relación del estilo de aprendizaje reflexivo con las teorías de enseñanza (tabla 14).

\begin{tabular}{|c|c|c|}
\hline Estilo de Aprendizaje & Análisis estadístico & Teoría de enseñanza \\
\hline \multirow{4}{*}{ Reflexivo } & $\mathrm{X}_{=}^{2}=.05$ & \multirow{4}{*}{ Dependiente o tradicional } \\
\hline & $\begin{array}{l}\text { Phi }=1.2 \\
\mathrm{U}=57\end{array}$ & \\
\hline & $\mathrm{Cc}=.79$ & \\
\hline & $\mathrm{X}^{2}==.05$ & \\
\hline \multirow[t]{3}{*}{ Reflexivo } & $\begin{array}{c}\mathrm{Phi}=1.3 \\
\mathrm{U}=.59\end{array}$ & \multirow[t]{2}{*}{ Productiva o tecnológica } \\
\hline & $\mathrm{Cc}=.79$ & \\
\hline & $\mathrm{X}^{2}==.05$ & \multirow{4}{*}{ Expresiva o activa } \\
\hline \multirow[t]{3}{*}{ Reflexivo } & $\begin{aligned} \text { Phi } & =.82 \\
\mathrm{U} & =.36\end{aligned}$ & \\
\hline & $\mathrm{Cc}=.63$ & \\
\hline & $\mathrm{X}^{2}==.05$ & \\
\hline \multirow[t]{3}{*}{ Reflexivo } & $\begin{array}{c}\mathrm{Phi}=1.2 \\
\mathrm{U}=.57\end{array}$ & \multirow[t]{2}{*}{ Interpretativa o constructivista } \\
\hline & $\mathrm{Cc}=.78$ & \\
\hline & $\mathrm{X}^{2}==-.05$ & \multirow{3}{*}{ Emancipadora o crítica } \\
\hline \multirow[t]{2}{*}{ Reflexivo } & $\begin{array}{c}\mathrm{Phi}=1.2 \\
\mathrm{U}=.55\end{array}$ & \\
\hline & $\mathrm{Cc}=.77$ & \\
\hline
\end{tabular}

Esta tabla muestra que el estilo de aprendizaje reflexivo no se relaciona con ninguna de las teorías de enseñanza.

Resultado de la relación del estilo de aprendizaje teórico con las teorías de enseñanza (tabla 15).

\begin{tabular}{|c|c|c|}
\hline Estilo de aprendizaje & Análisis estadístico & Teoría de enseñanza \\
\hline \multirow{4}{*}{ Teórico } & $\mathrm{X}^{2}=.05$ & \multirow{4}{*}{ Dependiente o tradicional } \\
\hline & $\mathrm{Phi}=1.3$ & \\
\hline & $\mathrm{U}=.62$ & \\
\hline & $\begin{aligned} \mathrm{Cc} & =.81 \\
\mathrm{X}^{2} & ==.05\end{aligned}$ & \\
\hline \multirow{3}{*}{ Teórico } & Phi $=1.4$ & \multirow{3}{*}{ Productiva o tecnológica } \\
\hline & $\mathrm{U}=.64$ & \\
\hline & $\mathrm{Cc}=.82$ & \\
\hline \multirow{5}{*}{ Teórico } & $\mathrm{X}^{2}=.001$ & \multirow{4}{*}{ Expresiva o activa } \\
\hline & Phi $=1.2$ & \\
\hline & $\mathrm{U}=.55$ & \\
\hline & $\mathrm{Cc}=.78$ & \\
\hline & $\mathrm{X}^{2}=. .05$ & \multirow{4}{*}{ Interpretativa o constructivista } \\
\hline \multirow{4}{*}{ Teórico } & Phi $=1.13$ & \\
\hline & $\mathrm{U}=.50$ & \\
\hline & $\mathrm{Cc}=.74$ & \\
\hline & $\mathrm{X}^{2}==.05$ & \multirow{3}{*}{ Emancipadora o crítica } \\
\hline \multirow[t]{2}{*}{ Teórico } & $\mathrm{Phi}=1.3$ & \\
\hline & $\begin{array}{l}U=60 \\
\mathrm{Cc}=80\end{array}$ & \\
\hline
\end{tabular}

Esta tabla muestra que el estilo de aprendizaje teórico solamente se relaciona con la teoría de enseñanza expresiva o activa.

Resultado de la relación del estilo de aprendizaje pragmático con las teorías de enseñanza (tabla 16).

Esta tabla muestra que el estilo de aprendizaje pragmático sólo se relaciona con la teoría de enseñanza emancipadora o crítica. 
Tabla 16 .

Tabla de contingencia que relaciona el estilo de aprendizaje pragmático con las teorías de enseñanza.

\begin{tabular}{|c|c|c|}
\hline Estilo de Aprendizaje & Análisis estadístico & Teoría de enseñanza \\
\hline \multirow{5}{*}{ Pragmático } & $\mathrm{X}^{2}=.05$ & \multirow{4}{*}{ Dependiente o tradicional } \\
\hline & $\mathrm{Ph}=1.3$ & \\
\hline & $\mathrm{U}=.69$ & \\
\hline & & \\
\hline & $\mathrm{X}^{2}==.05$ & \multirow{5}{*}{ Productiva o tecnológica } \\
\hline \multirow{4}{*}{ Pragmático } & Phi $=1$ & \\
\hline & $\mathrm{U}=.54$ & \\
\hline & $\mathrm{Cc}=.73$ & \\
\hline & $\mathrm{X}^{2}==.05$ & \\
\hline \multirow[t]{3}{*}{ Pragmático } & Phi $=.69$ & \multirow[t]{2}{*}{ Expresiva o activa } \\
\hline & $\begin{aligned} \mathrm{U} & =.35 \\
\mathrm{C}_{\mathrm{C}} & =557\end{aligned}$ & \\
\hline & $\mathrm{X}^{2}==.05$ & \multirow{4}{*}{ Interpretativa o constructivista } \\
\hline \multirow{3}{*}{ Pragmático } & Phi $=1.04$ & \\
\hline & $\mathrm{U}=.52$ & \\
\hline & $\mathrm{Cc}=.72$ & \\
\hline \multirow{4}{*}{ Pragmático } & $\mathrm{X}^{2}=.013$ & \multirow{4}{*}{ Emancipadora o crítica } \\
\hline & Phi $=1.45$ & \\
\hline & $\mathrm{U}=.72$ & \\
\hline & $\mathrm{Cc}=.82$ & \\
\hline
\end{tabular}

Análisis estadístico para conocer la relación que hay entre los Estilos de Aprendizaje y las Teorías de la Enseñanza, analizando los datos sin los ítems cinco, siete, 11 y 24

Resultado de la relación del estilo de aprendizaje activo con las teorías de enseñanza (tabla 17).

\begin{tabular}{|c|c|c|}
\hline Estilo de aprendizaje & Análisis estadístico & Teoría de enseñanza \\
\hline & $\mathrm{X}^{2}=.05$ & \\
\hline Activo & $\begin{array}{c}\mathrm{Phi}=1.3 \\
\mathrm{U}=.55 \\
\mathrm{Cc}=80\end{array}$ & $\begin{array}{l}\text { Dependiente o tradicional } \\
\text { (sin el ítem 5) }\end{array}$ \\
\hline & $\mathrm{X}^{2}==.05$ & \\
\hline Activo & $\begin{array}{l}\mathrm{Phi}=1.1 \\
\mathrm{U}=.45\end{array}$ & $\begin{array}{l}\text { Interpretativa o constructivista } \\
\text { (sin el ítem 11). }\end{array}$ \\
\hline & $\begin{aligned} \mathrm{C} c & =.74 \\
\mathrm{X}^{2} & ==05\end{aligned}$ & \\
\hline Activo & $\begin{array}{c}\text { Phi }=1.4 \\
\mathrm{U}=.58\end{array}$ & $\begin{array}{l}\text { Emancipadora o crítica } \\
\text { (sin los ítems } 7 \text { y } 24 \text { ) }\end{array}$ \\
\hline
\end{tabular}

Los resultados de esta tabla muestran que el estilo de aprendizaje activo no se relaciona con ninguna de las teorías de enseñanza.

Resultado de la relación del estilo de aprendizaje reflexivo con las teorías de enseñanza (tabla 18).

Tabla 18

Tabla de contingencia que relaciona el estilo de aprendizaje reflexivo y las teorías de enseñanza.

\begin{tabular}{ccc}
\hline Estilo de aprendizaje & Análisis estadístico & Teoría de enseñanza \\
\hline \multirow{3}{*}{ Reflexivo } & $\mathrm{X}^{2}=>.01$ & \\
& $\mathrm{Phi}=1.6$ & Dependiente o tradicional \\
& $\mathrm{U}=.71$ & (sin el ítem 5$)$ \\
$\mathrm{Cc}=.84$ & \\
Reflexivo & $\mathrm{X}^{2}=<.05$ & Interpretativa o constructivista \\
& $\mathrm{Phi}=.97$ & \\
& $\mathrm{U}=.43$ & \\
& $\mathrm{Cc}=.69$ & (sin el ítem 11$)$ \\
Reflexivo & $\mathrm{X}^{2}=<.05$ & Emancipadora o crítica \\
& $\mathrm{Phi}=1.3$ & \\
& $\mathrm{U}=.58$ & \\
$\mathrm{Cc}=.79$ &
\end{tabular}

Esta tabla muestra que el estilo de aprendizaje reflexivo sólo se relaciona con la teoría de enseñanza dependiente (o tradicional).

Resultado de la relación del estilo de aprendizaje teórico con las teorías de enseñanza (tabla 19).

\begin{tabular}{|c|c|c|}
\hline Estilo de aprendizaje & Análisis estadístico & Teoría de enseñanza \\
\hline \multirow{4}{*}{ Teórico } & $\mathrm{X}^{2}=<.05$ & \multirow{4}{*}{$\begin{array}{l}\text { Dependiente o tradicional } \\
\quad \text { (sin el ítem 5) }\end{array}$} \\
\hline & $\mathrm{Phi}=1.3$ & \\
\hline & $\mathrm{U}=.59$ & \\
\hline & $\mathrm{Cc}=.78$ & \\
\hline \multirow{5}{*}{ Teórico } & $\mathrm{X}^{2}=<.05$ & \multirow{5}{*}{$\begin{array}{l}\text { Interpretativa o constructivista } \\
\text { (sin el ítem 11) }\end{array}$} \\
\hline & Phi $=1.08$ & \\
\hline & $\mathrm{U}=.48$ & \\
\hline & $\mathrm{Cc}=.73$ & \\
\hline & $\mathrm{X}^{2}=<.05$ & \\
\hline \multirow{2}{*}{ Teórico } & Phi $=1.2$ & \multirow{2}{*}{$\begin{array}{l}\text { Emancipadora o crítica } \\
\text { (sin los ítems } 7 \text { y 24) }\end{array}$} \\
\hline & $\mathrm{U}=.55$ & \\
\hline
\end{tabular}

Esta tabla muestra que el estilo de aprendizaje teórico no se relaciona con ninguna teoría de enseñanza.

Resultado del estilo de aprendizaje pragmático con las teorías de enseñanza (tabla 20).

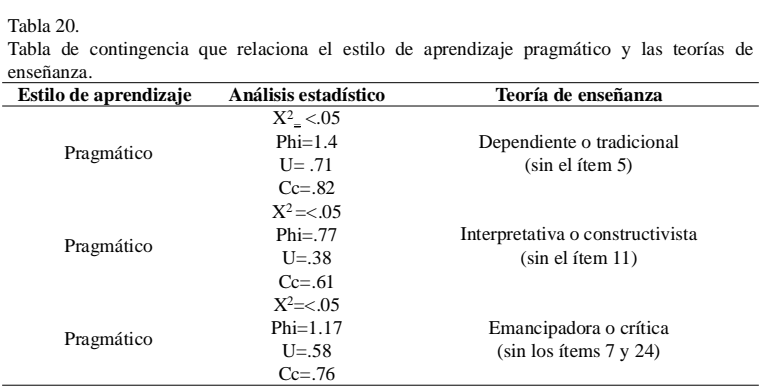

Esta tabla muestra que el estilo de aprendizaje pragmático no se relaciona con ninguna de las teorías de enseñanza.

\section{Discusión de resultados}

La mayoría de los estilos de aprendizaje se relacionan con las teorías de enseñanza: el estilo de aprendizaje activo se relaciona con la teoría de enseñanza tradicional, el teórico con la teoría de enseñanza expresiva o activa, el pragmático con la teoría de enseñanza emancipadora o crítica y el reflexivo no se relaciona con ninguna teoría de enseñanza. Estos resultados tienen que ver con que en cada salón de clase hay estudiantes que poseen diferentes estilos para aprender (aunque puede ser que predomine más un estilo -o más- que otro -s-) y de igual manera en los docentes, por tanto, siempre cabe la posibilidad de que las formas de enseñar de alguno o algunos de los maestros empaten con las diferentes formas de aprender de los estudiantes y viceversa. A continuación se describen algunos aspectos teóricos que fundamentan estos resultados.

La relación del estilo de aprendizaje activo con la teoría de enseñanza tradicional, indica que los alumnos activos no tienen ningún problema en implicarse en los programas y tareas planificadas por los maestros que tienen un estilo tradicional, porque les gusta trabajar en nuevas experiencias y desafíos, son de mente abierta y entusiasta, les gusta descubrir, arriesgarse e improvisar, son muy activos y retienen mejor la información discutiendo, explicando y aplicando el conocimiento, les gusta aprender desarrollando guías de estudio, carteleras, trabajos, talleres. $\mathrm{Y}$ aunque los docentes con un estilo de enseñanza tradicional no incluyen en su docencia todas estas características, tienen la ventaja de que los alumnos con las características de ese estilo (activo) tienen la posibilidad de adaptarse al estilo de enseñanza (tradicional), ya que poseen una mente abierta y se pueden adaptar a trabajar, ser dirigidos y controlados en cualquier forma (directa y autoritaria), y de acuerdo a las tareas que se les asignen de manera guiada, centrándose en el contenido del programa establecido de la materia, con una evaluación estandarizada y reflejada en una calificación(Marrero, 1993b, Sicilia \& Delgado, 2002; Bigge, 2007; Camaras, 2009), siendo éstas algunas de las características de la enseñanza tradicional.

El estilo de aprendizaje teórico se relaciona con la teoría de enseñanza expresiva o activa solamente en que algunas de las características de estas formas de aprender y de enseñar se adaptan durante el proceso educativo. Tal es el caso en el que los docentes programan algunas tareas mediante el descubrimiento guiado, enfatizando en la actividad, en la indagación, en la programación de contenidos variados lo cual empata con los alumnos teóricos que prefieren actividades objetivas, estructuradas y metódicas para aprender, obteniendo una evaluación de su rendimiento tanto cuantitativa como cualitativa. Pero no es lo mismo cuando los profesores (expresivos o activos) transmiten su enseñanza en forma global, ya que los alumnos teóricos aprenden mejor utilizando un pensamiento profundo, buscando, analizando, comprendiendo y construyendo teorías, principios y modelos coherentes y con una enseñanza global no tendrán los estímulos requeridos para lograr sus objetivos. Además, los docentes con este estilo de enseñanza (expresiva o activa) instruyen de manera práctica mientras que los alumnos teóricos son disciplinados, racionales, lógicos, críticos, tienden a la perfección y les gustan las clases magistrales. Esta información indica que aunque los resultados demuestren que hay una relación entre los estilos de aprender de los alumnos con las teorías de enseñanza de los profesores, ésta 
última no es completa ni integral (Honey \& Mumford, 1986; Alonso, Gallego \& Honey, 1994; Correa, 2006; Marrero, 1993a).

La relación del estilo de aprendizaje pragmático con la teoría de enseñanza emancipadora o crítica se presenta probablemente porque la mayor parte de las características que forman parte de ambos estilos se pueden ajustar. Por ejemplo a los docentes de estilo crítico les gusta programar contenidos que pueden ser aplicados en forma abierta y de acuerdo con las características del grupo y utilizan la técnica de la indagación mediante la investigación-acción. Esto beneficia a los alumnos de estilo pragmático porque la instrucción que reciben se ajusta a sus características, respetando sus ideas prácticas, seguras, funcionales y su aplicación y experimentación a la brevedad. Al permitir la aplicación de los contenidos en forma abierta, los estudiantes pueden descubrir, innovar y mejorar continuamente, aspectos todos que forman parte de sus preferencias para aprender. Como el docente interactúa con los estudiantes llevando a cabo una organización abierta y tratando el contenido temático como una problemática social y crítica, procurando que los alumnos aprendan descubriendo e interactuando con la cooperación de todo el grupo, esto permite que se dé una mayor y mejor comunicación entre ambos, estimulando otras características de los alumnos con este estilo (pragmático): son personas directas, realistas, decididas, planificadoras, visuales, rápidas para resolver problemas y les gusta trabajar apoyadas con material didáctico, (Honey \& Mumford, 1986; Alonso, et al, 1994; Correa, 2006 \& Marrero, 1993).

El estilo de aprendizaje reflexivo no se relaciona con ninguna de las teorías de enseñanza, es decir no todas las características y las preferencias de los alumnos reflexivos empatan con las características y con las preferencias de los docentes tradicionales, expresivos, tecnológicos, constructivistas y críticos. Tal vez esto se debe a que no todos los docentes incluyen en su instrucción estrategias, métodos y técnicas enfocadas a la investigación y a la acción como producto de esta, durante el proceso de enseñanza-aprendizaje, lo cual puede considerarse como el eje central de donde parten algunas de las características de este tipo de alumnos (reflexivos) porque ellos prefieren aprender observando las experiencias desde distintas perspectivas, de donde recogen los datos y los analizan antes de llegar a una conclusión, son prudentes les gusta considerar todas las alternativas posibles antes de realizar un movimiento, disfrutan escuchando y observando la actuación de los demás y no intervienen hasta que se han adueñado de la situación. Crean a su alrededor un aire ligeramente distante y condescendiente; son ponderados, concienzudos, receptivos, analíticos, asimiladores y exhaustivos (Honey \& Mumford, 1986 \& Alonso, et al, 1994); prefieren pensar detenidamente sobre el objeto de estudio y trabajar solos; son pacientes, aumentan la comprensión organizando la información en pasos lineales, pueden no entender el material, pero logran conectar lógicamente sus partes, prefieren la elaboración de mapas conceptuales, diagramas de flujo y árboles de problemas (Honey \& Mumford, 1986; Alonso, et al, 1994 \& Correa, 2006).

Los alumnos reflexivos, aprenden mejor si el docente planifica actividades donde ellos tengan la oportunidad de observar, pensar, analizar y comprender la tarea antes de actuar; donde tengan la oportunidad de trabajar solos, con tiempo suficiente, y utilizando estrategias que les permitan representar la información aprendida en forma clara, precisa y bien organizada. Pero les cuesta más trabajo aprender cuando tienen que realizar tareas donde ellos sean el centro de atención, o cuando se les apresura a realizar diferentes actividades o tienen que actuar sin haber realizado una planificación previamente (Díaz, 2012). Por tanto, no les gusta ser controlados ni presionados (características de la enseñanza tradicional), no les gusta hacer y tener la información en forma general (características de la enseñanza expresiva), no están de acuerdo en llevar programas organizados con contenidos abiertos y sí en aprender mediante la indagación-investigativa integrando el aprendizaje mediante el descubrimiento y la búsqueda de significados (características de la enseñanza constructivista), no conciben el conocimiento como una tradición cultural, como algo propio de la razón, como la recepción de información y la evaluación del rendimiento de manera estándar, cuantitativa e individualizada (características de la enseñanza productiva o tecnológi- ca) y no les interesa trabajar en grupo, ni programar y experimentar rápidamente (características de la enseñanza crítica).

La teoría de enseñanza tradicional es la preferida por los docentes y se relaciona con el estilo de aprendizaje activo que es el que más prefieren los alumnos. También empatan la teoría de enseñanza emancipatoria con el estilo de aprendizaje pragmático que son los menos preferidos por los profesores y por los estudiantes. El resultado de esta relación es importante ya que los profesores y los alumnos son protagonistas en el planteamiento, desarrollo y solución de las tareas manifestando durante el proceso sus características personales a través de sus estilos de enseñar, de aprender y de comunicarse (Ortiz \& De los Ángeles, 2007). Tal vez esta relación entre estilos predominantes se debe a que los alumnos de esta carrera son activos, de mente abierta, entusiastas, improvisadores, descubridores, arriesgados, espontáneos, participativos, les agradan las guías de estudio, trabajos, talleres y no son escépticos (Alonso, et al, 1994 \& Honey \& Mumford en 1986) y por tanto se adaptan a los profesores que programan los contenidos en forma lineal y técnica, les dan instrucciones directas e interaccionan con ellos mediante una clase organizada en forma controlada y usan estilos de enseñar reproductivos. Pero es importante que los profesores adapten y ajusten sus métodos de enseñanza para conseguir que todos sus alumnos se conecten con los objetivos del aprendizaje escolar despertando su interés y cuidando su bienestar y autoestima (Marchesi, 2004).

\section{Conclusiones}

En relación con los resultados obtenidos de esta investigación se sugiere diseñar instrumentos donde la totalidad de los ítems midan lo que realmente se estipula en los factores relacionados con las teorías de enseñanza y con los estilos de aprendizaje. Es importante integrar en el instrumento los aspectos que influyen y forman parte de la forma de enseñar y de aprender entre los cuales están: la historia de vida y el estado de salud de los profesores y de los estudiantes, los tipos de estrategias, métodos, técnicas y otros recursos didácticos personales que éstos conocen, utilizan o construyen; aspectos relacionados con la vocación, con los hábitos (para enseñar y aprender), con los valores morales, personales y éticos, con factores psicológicos (motivación, autoimagen, autoestima, seguridad, niveles de estrés, ansiedad, temor, indiferencia, gusto o disgusto, problemas emocionales, indiferencia), ambientales (preferentes y no preferentes) y contextuales (en los que están o no de acuerdo) de los docentes y educandos, con el fin de saber si estos factores influyen en las formas de enseñar y aprender, en la estabilidad emocional y en el rendimiento personal, académico y profesional; por ello se requiere construir, reconstruir o depurar los instrumentos para medir lo que se quiere con rigor estadístico, tomando en cuenta las bases teóricas de partida, las experiencias de docentes y estudiantes, los resultados de investigaciones sobre este tema, los factores, principios, teorías y demás recursos inherentes en la enseñanza y para el aprendizaje, para probar si los instrumentos diseñados son fiables y válidos. Estos últimos deberán ser revisados y aprobados por un comité de expertos, para aplicarlos posteriormente en una prueba piloto, de donde se obtengan los datos necesarios para dejarlos así o hacerles las adecuaciones requeridas y finalmente canalizarlos nuevamente al comité de expertos para su revisión y aprobación.

\section{Referencias}

Aguado, R., López, A., y Hernández, J.L., (2017). Educación Física y desarrollo de la autonomía: la percepción del alumnado de Educación Secundaria. Retos. 31, 300-305.

Alonso, C., Gallego, D. \& Honey, P. (1994). Los Estilos de Aprendizaje. Procedimientos de Diagnóstico y Mejora. España: Bilbao Mensajero.

Bahamón, M., Vianchá, M., Alarcón, L., \& Bohórquez, C. (2012). Estilos y estrategias de aprendizaje relacionados con el logro académico en estudiantes universitarios. Pensamiento Psicológico, 9(1), $115-12$. 
Ballesta, F., Izquierdo, T., y Romero, B. (2011). Percepción del alumnado de Pedagogía ante el uso de metodologías activas. Educación Siglo XXI, 2(29), 353-368.

Bigge, M. (2007).Teorías de Aprendizaje para Maestros. México. Trillas.

Camaras, R. (2009). «Estudio comparativo de los diferentes Estilos de Enseñanza en Educación Física». Revista Digital, Innovación y Experiencias Educativas. 18. Granada.

Correa, J. E. (2006). Identificación de los estilos de aprendizaje en los estudiantes de fisiología del ejercicio de la Facultad de Rehabilitación y Desarrollo Humano. Revista Ciencias de la Salud, 4, 41-53.

Chou, C., Bentler, P., \& Satorra, A. (1991). Scaled Test Statistics and Robust Standard Errors for-Non-normal Data in Covariance Structure Analysis: A Monte Carlo Study. British Journal of Mathematical and Statistical Psychology, 44 (Pt 2)(2):34757 - December 1991†with†77 Reads. DOI: 10.1111/j.20448317.1991.tb00966.x · Source: PubMed.

Delgado, M.A., y Zurita, F. (2003). Estudio de las Teorías Implícitas de la Educación Física en la formación inicial de los maestros en las diferentes especialidades de la Facultad de Ciencias dela Educación de la Universidad de Granada. Diferencias en función del Género. Retos. Nuevas tendencias en Educación Física, Deportey Recreación. 5, 27-38. Federación Española de Asociaciones de Docentes deEducación Física (FEADEF). ISSN edición impresa. 1579-1726.

Díaz, T.A. (2012). Informe de Investigación: «Relación entre los estilos de aprendizaje y el rendimiento académico de las estudiantes de la Escuela Profesional de Enfermería de la Facultad de Ciencias de la Salud de la Universidad Nacional de Callao». Universidad Nacional del Callao. Instituto de Investigación. Bellavista-Callao. Resolución Rectoral No. 356-2011-R.

Fernández, M., y Espada, M. (2017). Formación inicial y percepción del profesorado sobre los estilos de enseñanza en Educación Física. Retos. 31, 69-75.

García, J.L. (2006). Los Estilos de Aprendizaje y las Tecnologías de la información y la Comunicación en la Formación del Profesorado. Tesis doctoral. Dirigida por CatalinaAlonso García. Madrid. Pearsons Prentice Hall.

Gil, P., Contreras, O. P., Isabel, G., González, S., García, L., De Moya, M. D., y López, A. (2007). Estilos de aprendizaje de los estudiantes de magisterio: Especial. Profesorado, Revista de Currículum y formación de profesorado, 12(2), 1-19.

Hair, J. F., Black, W. C., Babin, B. J., Anderson, R. E., \& Tatham, R. L. (2006). Multivariate data analysis (Vol. 6). Upper Saddle River, NJ. Pearson Prentice Hall.

Honey \& Alonso, C. (1992). Cuestionario Honey-Alonso de Estilos de Aprendizaje (CHAEA). Recuperado de http:// www.estilosdeaprendizaje.es/ el 2 de Junio de 2016.

Honey, P. \& Mumford, A. (1986). The Manual of Learning Styles. P. Honey, Ardingly House, Maindenhead, Berkshire.

Hu, L., \& Bentler, P. M.(1999). Cutoff criteria for fitindexes in covariance structure analysis: conventional criteria versus new alternatives. Structural Equation Modeling, 6, 1-55.

Isaza, L. (2014). Estilos de Aprendizaje: una apuesta por el desempeño académico de los estudiantes en la Educación Superior. Revista Encuentros, Universidad Autónoma del Caribe, 12(2), 25-34.

Kidder, L., \& Judd, Ch. (1986). Research Methods In Social Relations, Fifth Edition. Holt Rinehart and Winston Inc. United States.

López, A., y Moreno, J. A. (2002). Aprendizaje de hechos y conceptos en educación física. Una propuesta metodológica. Apunts. Educación Física y Deportes, 63, 16-26.

Madrid, P. D., Prieto, A., Samalot, A., y Gil, P. (2016). Evaluación de una propuesta extraescolar de conductas apropiadas en educación física y deportiva. Retos. Nuevas tendencias en Educación Física, Deporte y Recreación 30, 36-42.

Marchesi, A. (2004). Qué será de nosotros, los malos alumnos. Madrid. Alianza.
Margalef, G (2014). Evaluación formativa de los aprendizajes en el contexto universitario: Resistencias y paradojas del profesorado. Educación XX1,17(2), 1-12.

Marrero, J. (1993a). Las teorías implícitas del profesorado: vínculos entre la cultura y la práctica de enseñanza. En Rodrigo, M. J, Rodríguez y Marrero, J(1993). Las teorías implícitas. Una aproximación al conocimiento cotidiano. «Diseño de la metodología para el estudio de las teorías implícitas». Madrid. Visor.

Marrero, J. (1993b). «Diseño de la metodología para el estudio de las teorías implícitas». Madrid. Visor.

Nunnally, J. C. (1970). Introduction to Psychological Measurement. New York: Mc Graw-Hill

Nuñez, J., y Reyes, C. (2014). La evaluación del aprendizaje de estudiantes: validación española del Assessment Experience Questionnaire (AEQ). ESTUDIOS SOBRE EDUCACIÓN, 26, 63-77.

Ortiz, E., y De los Ángeles, M. (2007). La psicodidáctica como enfoque interdisciplinario del proceso de enseñanza-aprendizaje universitario. Pedagogía Universitaria, 12(3), 32-45.

Richaud de M. (2005a). Articulación de la teoría psicológica y la teoría psicométrica [Articulation between psychologic theory and psychometric theroy]. Suma Psicológica, 12(1), 7-21.

Richaud, de M.(2005b). Desarrollos del análisis factorial para el estudio de ítems dicotómicos y ordinales. Interdisciplinaria, 22(2), 237251.

Rodrigo, M. J., Rodríguez A., y Marrero, J. (1993). Las Teorías Implícitas. Una aproximación al conocimiento cotidiano. Madrid. Visor. 246-7.

Serra, J., Muñoz, C. L., Cejudo, C., y Gil, P. (2017). Estilos de aprendizaje y rendimiento académico de universitarios de Educación Física chilenos. Retos.32, 62-67.

Shen, B., \& Chen, A. (2007). An examination of learning profiles in physical education. Journal of Teaching in Physical Education, 2(26), 145-160.

Sicilia, A., y Delgado, M. A. (2002). Educación Física y Estilos de enseñanza. España. INDE.

\section{Referencias electrónicas}

http://www.pwpamplona.com/wen/calcu/calculadora1.htm recuperado el 1. de Agosto de 2016. 